\title{
Croissance et revenu du travail agricole en Inde. Une économie politique de la divergence (1950-2014)
}

\begin{abstract}
Avertissement
Le contenu de ce site relève de la législation française sur la propriété intellectuelle et est la propriété exclusive de l'éditeur.

Les œuvres figurant sur ce site peuvent être consultées et reproduites sur un support papier ou numérique sous réserve qu'elles soient strictement réservées à un usage soit personnel, soit scientifique ou pédagogique excluant toute exploitation commerciale. La reproduction devra obligatoirement mentionner l'éditeur, le nom de la revue, l'auteur et la référence du document.

Toute autre reproduction est interdite sauf accord préalable de l'éditeur, en dehors des cas prévus par la législation en vigueur en France.
\end{abstract}

\section{revues.org}

Revues.org est un portail de revues en sciences humaines et sociales développé par le Cléo, Centre pour l'édition électronique ouverte (CNRS, EHESS, UP, UAPV).

Référence électronique

Bruno Dorin et Claire Aubron, « Croissance et revenu du travail agricole en Inde. Une économie politique de la divergence (1950-2014) », Économie rurale [En ligne], 352 | mars-avril 2016, mis en ligne le 01 avril 2018, consulté le 12 avril 2016. URL : http://economierurale.revues.org/4865

Éditeur : Société Française d'Économie rurale

http://economierurale.revues.org

http://www.revues.org

Document accessible en ligne sur : http://economierurale.revues.org/4865

Ce document est le fac-similé de l'édition papier.

Cet article a été téléchargé sur le portail Cairn (http://www.cairn.info).

\section{CAIRN}

Chercher. Repérer. Avancer.

Distribution électronique Cairn pour Société Française d'Économie rurale et pour Revues.org (Centre pour l'édition électronique ouverte)

(c) Tous droits réservés 


\title{
Croissance et revenu du travail agricole en Inde
}

\section{Une économie politique de la divergence (1950-2014)}

\author{
Bruno DORIN • CSH, New Delhi, Inde ; Cirad, Montpellier ; Cired, Nogent-sur-Marne \\ bruno.dorin@csh-delhi.com \\ Claire AUBRON • Montpellier SupAgro, UMR 868 Selmet, Montpellier ; ILRI, New Delhi, Inde \\ claire.aubron@supagro.fr
}

Malgré $7 \%$ de croissance économique annuelle moyenne en vingt ans contre 1,7\% pour la population, près de $55 \%$ des actifs indiens dépendent encore de l'agriculture en 2011. L'exposé, articulé sur trois niveaux d'analyse (comparaisons internationales depuis 1970, économie politique depuis I'Indépendance en 1947, diagnostic agraire microlocalisé en 2014), montre comment le sous-continent demeure piégé dans une trajectoire opposée au modèle canonique de croissance : la population active agricole augmente au lieu de régresser, de même que son écart de revenu moyen avec les autres actifs. La libéralisation économique a bien dopé la croissance non agricole, mais cette dernière s'avère bien trop pauvre en emplois. Ceci exacerbe une pression foncière $(0,65$ ha/actif en 2011) aggravée localement par d'inégaux accès à la terre, à l'eau et à la valeur ajoutée. Une telle transformation structurelle appelle des innovations institutionnelles et techniques adaptées à ces contextes typiquement asiatiques.

MOTS-CLÉS : Inde, agriculture, emploi, croissance, transformation structurelle

\section{Growth and agricultural labour productivity in India: a political economy of divergence (1950-2014)}

Despite an average annual economic growth of $7 \%$ over twenty years, as against a population growth of 1.7\% for the same period, in 2011 nearly 55\% of the Indian workforce is still dependent on agriculture. Our paper, based on three levels of analysis (international comparisons since 1970, political economy since independence in 1947, micro-located agrarian diagnosis in 2014), shows how the subcontinent remains trapped in a path diametrically opposite to the canonical model of growth. The agricultural workforce increases instead of decreasing, along with the labour productivity gap between farm and nonfarm workers. The economic reforms of 1991 boosted growth in the nonfarm sector but the latter generates few jobs. This exacerbates the land-labour ratio (0.65 ha per agricultural worker in 2011), aggravated locally by unequal access to land, water and value added. Such a structural transformation calls for institutional and technical innovations adapted to this typical Asian context.

KEYWORDS: India, Agriculture, Employment, Growth, Structural Transformation

$\mathbf{R}$ épublique fédérale de 29 États (et 7 territoires) et plus grande démocratie du monde en nombre d'habitants (plus de 1,2 milliard selon le recensement général de 2011, soit 382 hab. $/ \mathrm{km}^{2}$ ), l'Inde est connue pour son indépendance et l'originalité de ses choix politiques depuis son émancipation des Britanniques en 1947. Ces ambitions perdurent et en font un pays émergent au sens discuté par Piveteau et Rougier (2010), au même titre que son taux de croissance économique élevé depuis une libéralisation de l'économie engagée dès les années 1980. Cependant, malgré $7 \%$ de croissance 
annuelle moyenne du PIB en vingt ans (1992-2011) contre 1,7\% pour la population, le sous-continent compte au début des années 2010 encore un tiers de sa population sous le seuil de pauvreté extrême, soit un tiers aussi de la population mondiale vivant avec moins de 1,25 US\$ par jour en parité de pouvoir d'achat (PPP) selon la Banque mondiale. La majorité de ses femmes et enfants souffrent encore de sous-nutrition et les inégalités s'accroissent au sein de la population (Subramanian et Jayaraj, 2015), notamment dans l'accès à l'éducation et à la santé (Basole et Basu, 2015). L'agriculture, qui ne génère plus que $14 \%$ du PIB mais dont dépendent encore près de $55 \%$ des actifs (CSO, 2015), soit près de 600 millions de personnes avec leur famille (Datta et Sharma, 2010), est au cœur de ce paradoxe où fortes croissance et pauvreté cohabitent.

Ce paradoxe questionne la « transformation structurelle » (Chenery et Srinivasan, 1998) de l'économie indienne, autrement dit le « déversement » (Sauvy, 1980) de ses actifs agricoles dans des secteurs d'activité à meilleur revenu du travail. Ce déversement fut massif dans les pays industrialisés et apparaît, dans la littérature économique, comme le modèle à suivre pour résorber la pauvreté rurale et voir converger à long terme les économies et niveaux de vie. Quelle est l'avancée de l'Inde sur ce chemin de développement? Et peut-elle courir sur ce dernier pour rattraper à terme ceux qui l'ont pavé (théorie néoclassique de la convergence, synthétisée et discutée dans Rodrik, 2013) ?

Pour approfondir ces questions, notre exposé articule trois niveaux d'analyse. $\mathrm{La}$ première partie s'appuie sur des données macroéconomiques internationales depuis 1970 pour montrer que l'Inde, aux côtés de ses voisins d'Asie continentale, semble piégée dans une trajectoire opposée à celle suivie depuis plusieurs décennies par les pays de l'OCDE. La deuxième partie propose une économie politique de l'Inde depuis
1950 reliant croissance, agriculture et emploi, pour montrer qu'en dépit d'expérimentations variées et plutôt volontaristes, elle peine grandement à sortir ses campagnes de la pauvreté. Avant de conclure, la troisième partie, centrée sur l'échelle locale et les relations sociales, analyse les vicissitudes de la transition agraire dans deux cantons d'un État du Nord-Ouest érigé en modèle de croissance, le Gujarat.

\section{Du modèle canonique de croissance aux dissidences asiatiques}

\section{Le modèle canonique de croissance}

Après la flambée internationale des prix alimentaires de 2007-2008, de nombreux économistes agricoles et du développement ont dénoncé le manque d'intérêt porté à l'agriculture depuis le milieu des années 1980, autant par la communauté académique que par celle des grands bailleurs de fonds (Janvry, 2010). On aurait oublié l'importance de l'agriculture dans le processus de croissance et de développement (FAO, 2009) pourtant depuis longtemps soulignée, notamment dans une vaste littérature d'après-guerre qui a forgé un véritable paradigme, celui de la «transformation structurelle» (Johnston et Mellor, 1961 ; Chenery et Srinivasan, 1998 ; Herrendorf et al., 2014). Ce paradigme s'est construit sur des conceptions et mesures de dynamiques économiques selon trois secteurs (primaire, secondaire et tertiaire: Fisher A.G.B., Clark C., Fourastié J.), des analyses de «croissance économique moderne » (Kuznets, 1966) de pays dits aujourd'hui développés ou industrialisés (les pays de l'OCDE), ou encore des modèles économiques duaux interdépendants comme celui de Lewis (1954) avec un secteur « de subsistance » (ailleurs : « traditionnel », « agricole », « rural », « informel», etc.) et un secteur «capitaliste» (ailleurs : « moderne », « non agricole», « urbain », « formel », etc.). 
Il en émane un modèle de développement où l'agriculture fournit une main-d'œuvre bon marché au processus d'urbanisation et d'industrialisation, laquelle livre en retour des technologies et des intrants industriels permettant d'augmenter la productivité de la terre et d'alimenter les populations à bas prix. Dans ce processus, la productivité du travail agricole augmente jusqu'à éradiquer la pauvreté rurale, et les dépenses d'alimentation des ménages diminuent fortement en pourcentage (loi de Engel) tout en accordant une part plus large aux produits transformés par l'industrie agroalimentaire. Ceci jusqu'à arriver dans le «monde sans agriculture» (Timmer, 2009) des pays aujourd'hui riches ; monde où cette dernière ne représente en effet plus que 2-3\% du PIB et des emplois, tandis qu'ont convergé les revenus moyens du travail agricole et non agricole.

Cette croissance canonique peut être caractérisée par deux critères délimitant un sentier idéal que nous avons ailleurs baptisé « chemin de Lewis » ou Lewis Path (LP) (Dorin et al., 2013) $)^{1}$ : (1) la population active agricole diminue en absolu (et pas seulement en pourcentage $)^{2}$; (2) les

1. Dans le modèle iconique de Lewis car d'économie duale (contrairement aux modèles de croissance dominants : Gollin, 2014), ce « Lewis Path » (caractérisé par nous, non par Lewis) pourrait être assimilé au transfert des actifs agricoles et de l'agriculture dans le secteur « capitaliste » où la productivité du travail est plus élevée « car fructifiée par le capital », i.e. hors du secteur « de subsistance » que Lewis caractérise par une main d'œuvre « illimitée » car à productivité marginale du travail négligeable voire négative, ceci en raison d'une surpopulation relativement aux ressources naturelles.

2. Ce point est fondamental dans notre démonstration. Le pourcentage d'actifs agricoles peut en effet diminuer (croissance plus importante de l'emploi dans les autres secteurs) mais bien peu réalisent que le nombre d'actifs agricoles peut parallèlement stagner voire augmenter, ce qui, comme nous le montrerons par exemple ici, a des conséquences dramatiques sur la croissance de la disponibilité par actif en capital-terre. productivités moyennes du travail agricole et non agricole convergent. Le croisement de ces deux critères permet de distinguer trois autres types d'évolution :

- dans la croissance agri-développante ou Farmer Developing (FD), il y a convergence des revenus mais augmentation du nombre d'actifs agricoles ;

- dans la croissance agri-excluante ou Farmer Excluding (FE), les agriculteurs sont de moins en moins nombreux et de plus en plus pauvres par rapport au reste de la population ;

- enfin, dans le piège ou la «trappe de Lewis » ou Lewis Trap (LP), à l'opposé du chemin de Lewis, le nombre d'actifs agricoles augmente tout comme leur écart de revenu avec les autres actifs.

Cette typologie va nous permettre de montrer que la trajectoire de développement des pays d'Asie continentale s'éloigne pour l'heure fortement du sentier canonique de transformation structurelle alors que de très nombreux travaux se convainquent du contraire, notamment parce qu'ils se limitent à observer la (réelle) décroissance en pourcentage de l'agriculture dans le PIB et la population active (ex. : Lin, 2011). Après cette démonstration (section suivante) ${ }^{3}$, toute la suite de notre exposé s'attachera à préciser les raisons et impacts socio-économiques et structurels d'une telle trajectoire en Inde.

\section{L'Asie continentale piégée dans l'agriculture de subsistance}

D'après nos estimations (Dorin et al., 2013), les nations embarquées sur le chemin de Lewis depuis 1970 (année où peuvent remonter les calculs pour la quasi-totalité des pays du monde, notamment de valeur ajoutée par actif) représentent

3. Pour une discussion approfondie de cette démonstration, tant au plan empirique que théorique, voir Dorin et al. (2013) et publication à venir. 


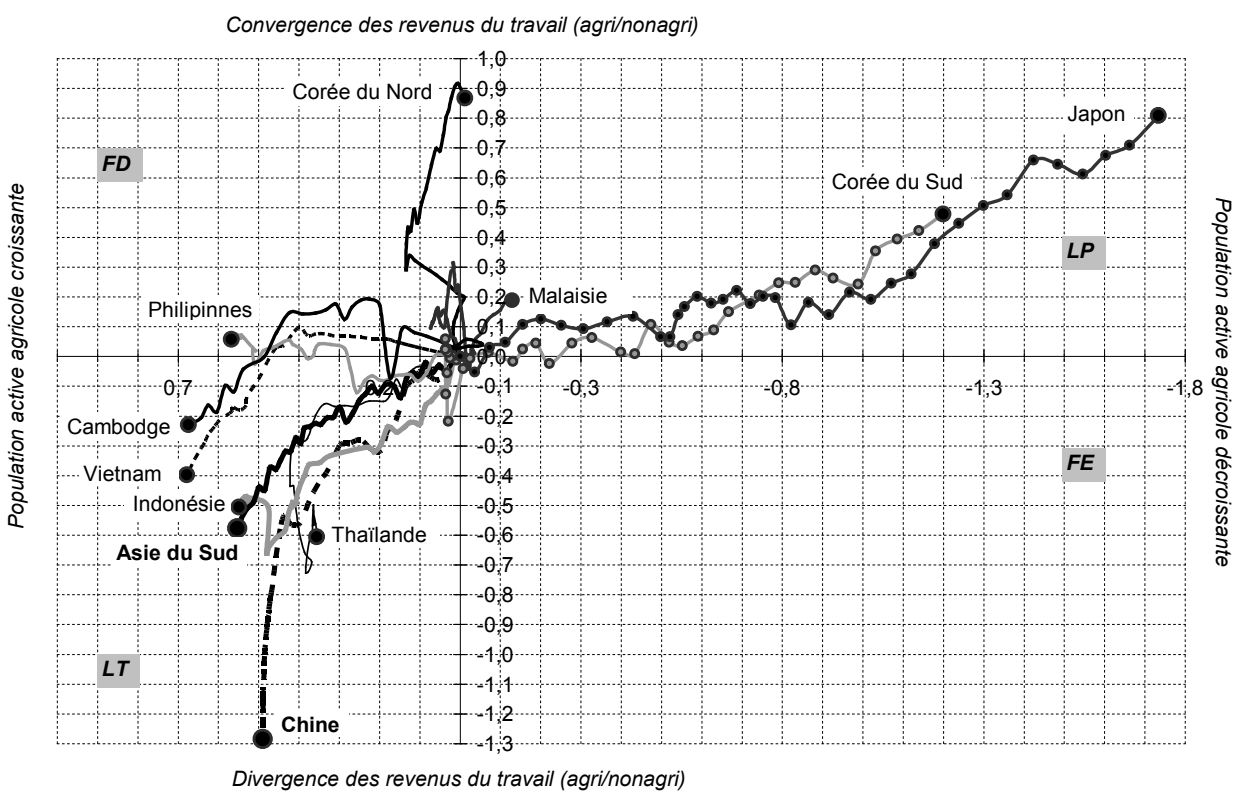

Source : d'après Dorin et al. (2013), avec données UNSTAT (2010) et FAO (2010).

$29 \%$ de la population mondiale de 2007. Il s'agit en très grande majorité des pays de l'OCDE ou en transition. La plupart des pays d'Amérique latine, d'Afrique et du Moyen-Orient suivent quant à eux - et pour l'heure - plutôt la voie agri-développante (FD), soit $16 \%$ de la population de 2007, alors que plus de la moitié de l'humanité $(55 \%)$, concentrée en Asie continentale, paraît engouffrée dans le piège de Lewis (LP).

La figure 1 représente des dynamiques nationales ou régionales de transformation en Asie en incluant les deux pays OCDE que sont le Japon et la Corée du Sud, tandis que la figure 2 zoome sur les pays d'Asie du Sud où s'inscrit l'Inde. Sur chaque axe des figures sont cumulés des taux annuels de croissance depuis 1970 (au centre), d'une part (abscisses) des populations actives agricoles telles que renseignées par la FAO (2010), d'autre part (ordonnées) d'un indicateur d'écart de revenu moyen entre travail agricole et non agricole $^{4}$ utilisant les valeurs ajoutées (agricoles et non agricoles) renseignées dans UNSTAT (2010) en dollar de 1990.

Avec ces figures, il apparaît que la trajectoire de l'Inde, à l'antipode du canon de transformation structurelle tout comme chez ses voisins asiatiques, présente deux grandes caractéristiques :

(1) une temporalité d'évolution similaire à la Chine (figure 1), laquelle se démarque néanmoins ${ }^{5}$ par une moindre

4. Nous appelons LIR ou Labour Income Ratio le rapport de la part de l'agriculture dans le PIB sur la part de l'agriculture dans les emplois (voir Dorin et al., 2013, pour plus de précisions, y compris sur données). Par rapport à l'APG (Agricultural Productivity Gap) utilisé par exemple dans Gollin et al. (2014), notre LIR présente l'avantage de normaliser les écarts entre 0 et 1 et de ne pas surestimer les inégalités quand ces écarts sont importants mais concernent, d'un côté ou de l'autre (actifs agricoles ou non agricoles), une faible part de la population.

5. Si ses statistiques sont fiables notamment en termes de population active. 


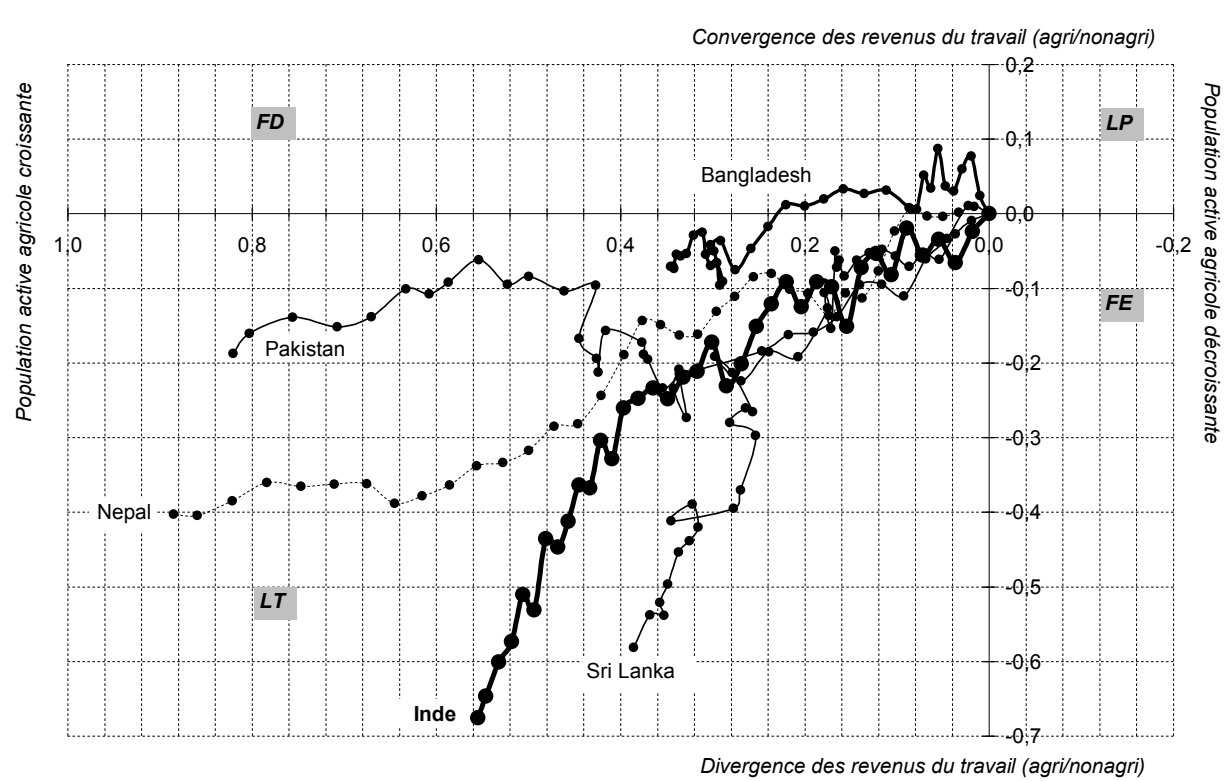

Note : les deux axes cumulent année après année les taux annuels de croissance observés depuis 1970 (au centre) des populations actives agricoles (en abscisses, avec une échelle inversée) et des écarts de revenu entre travail agricole et non agricole (en ordonnées) ; ces axes délimitent quatre grandes trajectoires d'évolution appelées Lewis Trap (LT), Farmer Developing (FD), Lewis Path (LP) et Farmer Excluding (FE).

Sources : d'après Dorin et al. (2013), avec données UNSTAT (2010) et FAO (2010).

croissance de la population active agricole qu'en Inde, mais un creusement plus important des écarts entre revenu du travail agricole et non agricole ;

(2) un cheminement en deux périodes distinctes (figure 2) avec, depuis 1993, une population active agricole qui tend à croître moins vite et un écart de revenu du travail qui au contraire se creuse plus rapidement. La charnière entre ces deux périodes coïncide avec l'accélération de la libéralisation économique du pays, comme nous allons l'étudier plus finement ci-après.

\section{Du socialisme nehruvien}

\section{à I'Inde qui brille : des campagnes toujours piégées}

Pour analyser le chemin de croissance économique de l'Inde indépendante et la contribution de l'agriculture à cette croissance, reflet de diverses stratégies pour moderniser le pays et sortir ses campagnes de la pauvreté, nous adoptons une démarche d'économie politique et distinguons cinq grandes phases. Cette distinction s'appuie sur des «moments critiques » et les rythmes différents d'évolution qu'ils insufflent, comme le montrent les courbes ou moyennes présentées ci-après (figure 3, tableaux 1 et 2) que la suite de l'exposé s'emploie à expliciter.

\section{L'industrie industrialisante du socialisme nehruvien (1950-1966)}

Vers le IV siècle avant J.-C., Kautilya, ministre du roi Chandragupta Maurya, posait dans son Traité du profit (Artha Shastra) la question des relations ambiguës entre artha et dharma, entre économie et politique. Il en sera de même en 1947, et les 


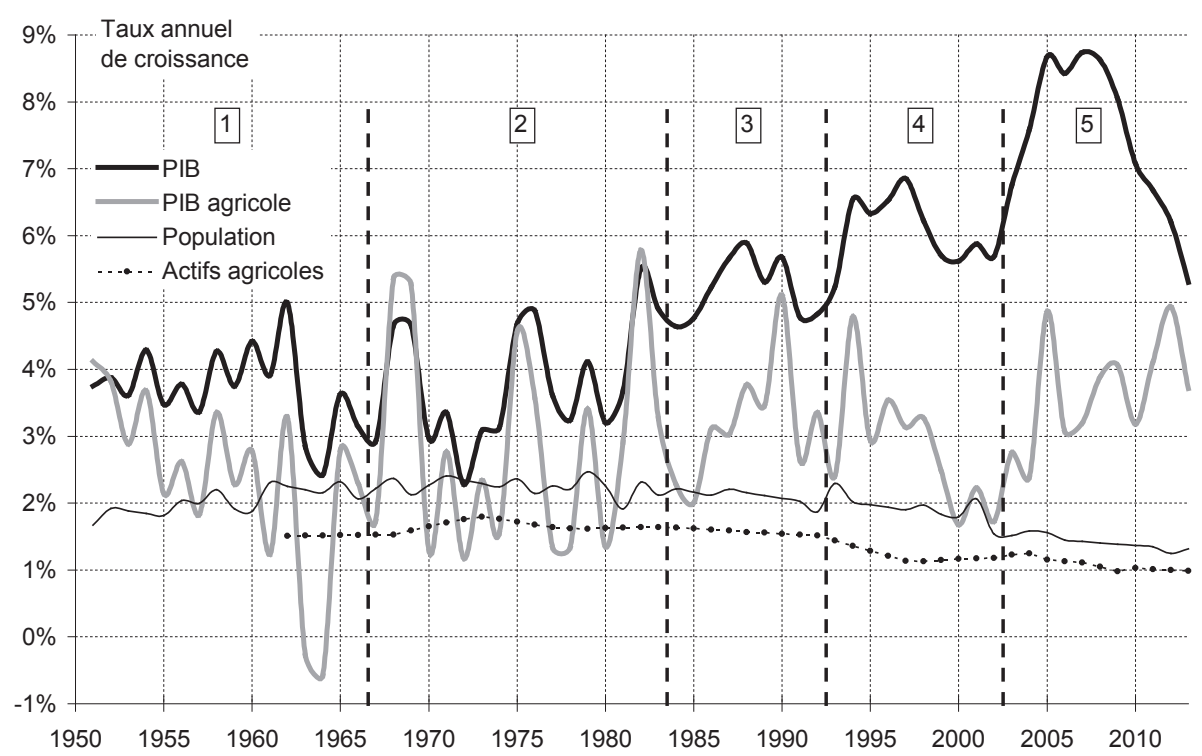

Notes: (1) PIB au coût des facteurs, à prix constant base 2004-05 (2) moyennes quinquennales mobiles pour les courbes de la figure (moyennes classiques dans les tableaux ci-après).

Source des données (figure et tableaux ci-après): CSO (2015) sauf actifs agricoles (Faostat, mars 2015).

Tableau 1. Taux annuels moyens de croissances économique et démographique (1950/51-2013/14)

\begin{tabular}{cccccccc}
\hline & & PIB & Population & PIB/hab & PIB $_{\text {agri }}$ & Actifs $_{\text {agri }}$ & PIB agri $_{\text {Actif }}$ agri \\
\hline 1 & $1950 / 51-1966 / 67$ & $+3,4 \%$ & $+2,0 \%$ & $+1,4 \%$ & $+1,8 \%$ & n.d. & n.d. \\
2 & $1967 / 68-1983 / 84$ & $+4,1 \%$ & $+2,3 \%$ & $+1,8 \%$ & $+3,6 \%$ & $+1,7 \%$ & $+1,9 \%$ \\
3 & $1984 / 85-1992 / 93$ & $+4,9 \%$ & $+2,1 \%$ & $+2,8 \%$ & $+2,8 \%$ & $+1,6 \%$ & $+1,2 \%$ \\
4 & $1993 / 94-2002 / 03$ & $+6,0 \%$ & $+1,9 \%$ & $+4,0 \%$ & $+2,3 \%$ & $+1,2 \%$ & $+1,1 \%$ \\
5 & $2003 / 04-2013 / 14$ & $+7,6 \%$ & $+1,4 \%$ & $+6,1 \%$ & $+4,1 \%$ & $+1,1 \%$ & $+3,0 \%$ \\
\hline
\end{tabular}

Source : les auteurs d'après CSO (2015) et Faosta (mars 2015).

Tableau 2. Part moyenne des trois grands secteurs économiques dans le PIB (1950/51-2013/14)

\begin{tabular}{ccccc}
\hline & & Agriculture & Industrie & Services \\
\hline 1 & $1950 / 51-1966 / 67$ & $47 \%$ & $14 \%$ & $37 \%$ \\
2 & $1967 / 68-1983 / 84$ & $38 \%$ & $17 \%$ & $43 \%$ \\
3 & $1984 / 85-1992 / 93$ & $31 \%$ & $20 \%$ & $49 \%$ \\
4 & $1993 / 94-2002 / 03$ & $24 \%$ & $21 \%$ & $54 \%$ \\
5 & $2003 / 04-2013 / 14$ & $16 \%$ & $20 \%$ & $64 \%$ \\
\hline
\end{tabular}

Source : les auteurs d'après CSO (2015) et Faosta (mars 2015). 
intellectuels qui dominent le parti du Congress s'entendent non pas sur un objectif de croissance économique, mais sur une dogmatique à double entrée (Dorin et Landy, 2002). La première, d'ambition mondiale, est d'affirmer l'indépendance nationale par un modèle original de développement : la fameuse troisième voie, ou encore le non-alignement. La seconde, déclinaison de la première au plan intérieur, est l'instauration d'une économie mixte où l'État intervient plus ou moins vigoureusement sur les marchés pour servir un objectif officiellement souverain : l'équité sociale dont dépend l'unité d'une Nation en complexe construction avec ses identités multiples et prononcées de religion, de caste, de région, de langue... Cet interventionnisme au nom de la lutte contre la concentration des richesses, les spéculateurs, les usuriers ou encore les monopoles fera paradoxalement aussi l'affaire de grands groupes industriels familiaux (Bajaj, Birla, Godrej ou Tata) formés dès le XIX ${ }^{\mathrm{e}}$ siècle (Markovits, 2014), puisqu'il leur permettra de protéger et développer un marché intérieur (Dorin, 2003). Jusqu'à la fin des années 1970, ce capitalisme d'État s'affirme et s'exprime sur bien des plans : industrialisation et planification quinquennale du développement, barrières aux importations et aux capitaux étrangers, nationalisations de certaines branches d'activité comme la banque, production sous licences et contingentements dans d'autres domaines (agroalimentaire, textile...) afin de multiplier, à côté de quelques poids lourds industriels, petites industries et petits commerces générateurs d'emplois.

Dans la première sous-période que nous distinguons ici s'institutionnalise le fameux «taux de croissance hindou » de $3,5 \%$. L'effort d'investissement est

6. Le Hindu rate of growth désignera le faible taux de croissance de l'économie planifiée indienne jusqu'aux réformes de 1991. porté sur l'industrie, ce qui conduit la croissance agricole à décliner $(+1,8 \%$ par an en moyenne, $-11 \%$ en 1965 aggravé par une sécheresse) et son écart avec le reste de l'économie à augmenter alors que la population croit annuellement de $+2 \%$ (figure 3, tableau 1).

Après le $\mathrm{I}^{\mathrm{er}}$ plan quinquennal d'avril 1951 à mars 1956 (1951-1955) ${ }^{7}$, plan d'inspiration keynésienne attaché à consolider les efforts de réorganisation entrepris depuis l'Indépendance (rare plan qui atteindra ses objectifs), les deux plans qui succèdent misent en effet sur l'industrie lourde pour permettre, d'une part une rapide substitution des importations, d'autre part l'indépendance à terme en biens d'équipement essentiels. Ce «syndrome soviétique » ou « schéma marxiste » est cependant indianisé $^{8}$. Dès 1955, Mahalanobis s'émancipe de la modélisation du soviétique Feldmann pour mieux représenter les dynamiques intersectorielles de consommation et d'emploi en Inde. Les phénomènes monétaires sont par contre ignorés. Pour le III ${ }^{\mathrm{e}}$ plan (1961-1965), Chakravarty se rapproche davantage encore de la structure économique indienne, mais le cadre général demeure celui d'une économie fermée, notamment aux investissements étrangers.

Dans les II ${ }^{\mathrm{e}}$ et $\mathrm{III}^{\mathrm{e}}$ plans, l'agriculture (y compris l'irrigation) ne représente finalement que $21 \%$ des dépenses totales. Elle n'est cependant pas délaissée compte tenu de son importance dans l'économie et l'emploi. Le souvenir de la famine de 1943, avec ses 4 millions de victimes directes et indirectes, n'est pas non plus éradiqué. Pour Nehru néanmoins, après la campagne

7. La plupart des statistiques indiennes, y compris agricoles, se rapportent à cette période d'avril à mars. Dans la suite du document, par commodité, nous ne mentionnons que la première année concernée.

8. On notera au passage que les deux principaux ouvrages de Lewis, publiés en 1949 puis 1966, portèrent sur la planification (Gollin, 2014). 
« Grow More Food» de 1943 à 1951, l'essor des campagnes repose davantage sur des changements structurels ou institutionnels : réformes agraires (première vague) ${ }^{9}$, coopératives de crédit villageois (Primary Agricultural Credit Societies, 1951) et développement communautaire (Community Development Programme, 1952). Mais comme dans d'autres domaines (droit du travail, lutte contre la violence de castes...), si des programmes ou lois sont décrétés, les moyens ne sont pas forcément mobilisés pour réellement les mettre en œuvre face aux intérêts des catégories dominantes (Appu, 1997 ; Pouchepadass, 2006). De nombreux travailleurs agricoles, exclus du processus de réforme agraire, restent ainsi sans terre et travaillent comme ouvriers agricoles à temps partiel ou complet (Breman, 2007a). Enfin, c'est aussi en 1956 qu'est signé l'accord avec les ÉtatsUnis qui, dans le cadre de la Public Law 480 (PL-480), conduit l'Inde à importer des surplus alimentaires américains (principalement grains ou farines de blé et riz), à bas prix et en roupies que les États-Unis utiliseront localement pour y affirmer leur influence technique et politique.

\section{La Révolution verte du blé et du riz (1967-1983)}

La croissance agricole reprend nettement de 1967 à 1983 pour suivre pratiquement celle du PIB d'environ $+4 \%$ par an tout au long de cette période (figure 3, tableau 1).

Cette reprise est engagée après une conjonction de crises qui reporteront à 1969 le début du VI ${ }^{\mathrm{e}}$ plan quinquennal : chômage croissant (la force de travail en

9. Une série de réformes agraires confiées aux États tentera, au fil du temps et suivant les régions, d'abolir le système zamindari, d'obliger la culture des terres possédées, de limiter l'achat de terres, de sécuriser bailleurs ou métayers, de faciliter le rachat de terre par le tenancier, de redistribuer aux paysans sans terre des biens confisqués ou des friches gouvernementales, etc. augmentation est peu absorbée par l'industrie « organisée » à forte intensité capitalistique), augmentation des dépenses d'armement (guerre avec la Chine en 1962, avec le Pakistan en 1965), mort de Nehru après l'humiliante défaite face à la Chine (1964), épuisement des stocks publics de grains suite à des moussons médiocres, dépendance politique croissante à l'aide américaine (PL-480), spéculation sur les prix et forte inflation (jusqu'à la dévaluation en juin 1966), apparition de troubles voire de famines dans certaines régions, récoltes catastrophiques de 1965 et $1966 . .$. L'Inde s'engage alors résolument dans ce qui deviendra la fameuse «Révolution verte » (Dorin et Landy, 2002) et, dans le IV ${ }^{\mathrm{e}}$ plan (1969-1973), elle reconnaît successivement la nécessité de mieux soutenir, d'une part les activités consommatrices de maind'œuvre, notamment celles des petites industries en zones rurales (la Khadi and Village Industries Commission, d'inspiration gandhienne, était née en 1956), d'autre part l'agriculture, qui doit assurer l'alimentation de base de la population mais aussi générer l'épargne nécessaire au financement des investissements publics. En 1969, 14 grandes banques de dépôt sont nationalisées ( 6 autres le seront avant 1980). Le secteur bancaire commercial est ainsi transformé en un véritable monopole d'État sous l'égide de la Banque nationale de l'agriculture et du développement rural (NABARD). De 1968 à 1977, le nombre d'agences en milieu rural est multiplié par six et le montant des dépôts par cinq.

Cette mobilisation de la petite épargne domestique aidera le développement de la Révolution verte après des prêts des fondations Ford et Rockefeller dès les années 1950. Les composantes techniques de cette révolution centrée sur le blé et le riz, et sur les régions fertiles irriguées ou pouvant l'être facilement, sont aujourd'hui bien connues. Il s'agit d'un paquet à trois ingrédients, avec des semences à haut rendement (importées 
à l'origine du CIMMYT au Mexique pour le blé, de l'IRRI aux Philippines pour le riz), des engrais chimiques et, avant tout, de l'irrigation (essentiellement par forages) non seulement pour que s'exprime le potentiel des nouvelles semences, mais aussi pour augmenter le nombre de cultures par an (l'«intensité culturale», souci central de la recherche agronomique indienne). La diffusion à grande échelle d'un tel paquet n'aurait pu se faire sans un complexe système d'incitations en amont et en aval. En amont, ce sont la recherche (sous l'égide de l'ICAR: Raina, 2011) et les subventions aux intrants, autant pour leur fabrication nationale (cas surtout des engrais) que pour leur utilisation par les producteurs (pompes, carburant ou électricité pour l'irrigation, engrais, semences). En aval, ce sont des prix d'achat attractifs pour les producteurs (Procurement Prices) qui permettent de constituer des stocks publics de blé et de riz que la Corporation alimentaire de l'Inde (FCI, née en 1964) est chargée de redistribuer à prix réduit dans tout le pays aux populations les plus démunies ${ }^{10}$, via le Système public de distribution (PDS) et ses centaines de milliers de boutiques « à juste prix » (FPS - Fair Price Shops).

C'est ainsi que l'Union indienne devient autosuffisante en blé et riz dès la fin des années 1970 (et même exportatrice plus tard), ainsi qu'en sucre de canne assez pareillement encouragé (Dorin et Landy, 2002). Cette concentration de l'intervention publique sur la production mais aussi l'accès (Sen, 1981) de ces trois denrées éloignera définitivement le spectre de grande famine en Inde. Mais elle induit en retour un délaissement de la production et de l'accès à d'autres denrées pourtant essentielles tant aux revenus qu'à l'alimentation équilibrée de nombreuses populations rurales. La sous-nutrition perdure (en protéines,

10. Les critères d'attribution des cartes de rationnement et les quotas par ménage ne cesseront d'être discutés et réformés au fil des décennies. acides gras essentiels, micronutriments...) tout comme la pauvreté rurale, notamment dans les zones arides ou semi-arides où les possibilités d'irrigation réduites ne permettent guère de profiter des ingrédients de la Révolution verte (Dorin et Jullien, 2004). Dès la fin des années 1970 sont alors aussi lancés une multitude de programmes anti-pauvreté rurale. Parmi les plus emblématiques figurent l'ICDS (Integrated Child Development Services, 1975) et le MDMP (Mid-Day Meal Program) pour améliorer la nutrition et le soin des enfants, l'IRDP (Integrated Rural Development Programme, 1978), le TRYSEM (Training of Rural Youth for Self-Employment, 1979) et le DWCRA (Development of Women and Children in Rural Areas, 1982) pour stimuler l'entrepreneuriat (Dorin et Landy, 2002 ; IDFC, 2013).

\section{Le grand tournant libéral (1984-1992)}

Les années 1980 marquent un grand tournant à plus d'un titre. Le taux de croissance démographique commence d'abord à décliner, et celui du PIB se hisse de plus en plus fermement au-dessus de $5 \%$ ( $f$ gure 3, tableaux 1 et 2) tandis que les subventions à la Révolution verte s'affirment (Dorin et Jullien, 2004) alors que s'essoufflent ses gains de productivité (Dorin et al., 2001) ${ }^{11}$.

Cette période amorce le déclin du Licence Raj qui contrôle et contingente les entreprises pour limiter leur taille et multiplier leur nombre afin de démultiplier

11. Les autres révolutions agricoles indiennes (lait, oléagineux, aviculture, aquaculture, etc.) s'affirment durant ces années 1980 (Dorin et Landy, 2002), suite aux quasi-stagnations de production et aux baisses de disponibilité par habitant que la Révolution verte aura quelque part engendrées pour bien des denrées. Mais le soutien public à ces révolutions (parfois temporaires comme pour les oléagineux) sera bien plus modeste voire quasi inexistant ; elles ont plutôt été tirées par la montée des prix et la demande croissante de la classe moyenne urbaine. 
l'emploi de main-d'œuvre, directement ou indirectement (intermédiaires, transporteurs, fonctionnaires...). Rajiv Gandhi sera le principal ordonnateur de ce changement lorsqu'il arrive au pouvoir après l'assassinat de sa mère Indira en $1984^{12}$. Quand il prend ses fonctions, le toutDelhi administratif est saisi d'une activité fébrile. Si «Garibi Hatao desh bachavo » («Éradiquer la pauvreté sauver le pays ») était la devise de sa mère, «India in the 21st century» («l'Inde dans le $\mathrm{XXI}^{\mathrm{e}}$ siècle ») est celle du nouveau jeune Premier ministre. Rajiv impressionne l'opinion et son parti par une autorité insoupçonnée, mais aussi par un style, celui d'un manager qui veut travailler plus vite, avec moins de bureaucratie, plus d'efficacité, d'honnêteté, de modernité, d'ouverture au monde. Derrière lui s'affirme également une classe moyenne issue du « socialisme à l'indienne » : paysans bénéficiaires de la Révolution verte, patrons d'entreprises familiales, cadres ou dirigeants des grandes sociétés, commerçants, fonctionnaires ou ouvriers protégés par de puissants syndicats. Cette classe grossit avec un désir, consommer, et une frustration, ne pas en avoir le pouvoir, étouffée qu'elle est par les dogmes tant gandhien (la croissance du bien-être matériel se traduit par une spoliation des plus nombreux au profit de quelques-uns) que nehruvien (privilégier l'effort d'épargne au détriment de la consommation pour dégager une forte capacité d'investissements publics). C'est sur cette «Inde de Lakshmi » (déesse de la fortune, de la beauté et de l'élégance) (Rudolph et Rudolph, 1987) que Rajiv compte pour dynamiser le secteur privé et le décorseter des routines et lourdeurs de l'administration. Dans le VII ${ }^{\mathrm{e}}$ plan

12. Lui-même assassiné en 1991 en pleine campagne électorale, cette décennie marque également la fin de l'hégémonie au pouvoir du parti du Congress, et annonce une ère de coalitions marquée par une succession accélérée de Premiers ministres.
(1985-1989), on envisage pour la première fois que ce secteur privé fournisse plus de $50 \%$ des investissements. Et les barrières aux importations sont abaissées pour démultiplier la fabrication nationale de ventilateurs, de mixers, de réfrigérateurs, de scooters, de téléviseurs et de multiples autres biens dont la consommation explose alors.

Mais la balance commerciale en souffre. Le déficit record après la seconde crise pétrolière de 1979 ne se résorbe guère durant toutes ces années 1980, d'autant que d'autres postes d'importation ont tendance à augmenter (armement, engrais, huiles alimentaires...) et que le pays ne développe toujours pas de fers de lance à l'exportation. À cela s'ajoute le coût croissant des ressources financières extérieures, puisqu'avec l'ouverture à l'international de la Chine, de certains Pays moins avancés et enfin des pays d'ex-URSS, l'aide étrangère et les crédits concessionnels se raréfient. Le service de la dette s'élève donc, et l'endettement tant interne (entreprises publiques) qu'externe (entreprises privées) qui en résultera conduit rapidement à la faillite financière de l'ensemble du pays. En juillet 1991, l'Inde doit gager pour la première fois une partie de son stock d'or à la Banque d'Angleterre. Elle perçoit ensuite un premier crédit de 8,7 milliards de dollars de la Banque mondiale et du Fonds monétaire international. Ce n'est pas la première fois que ce dernier prête à l'Inde (6 milliards en 1981), mais cette fois-ci, il n'est plus question de ne pas se plier aux recommandations des bailleurs de fonds, c'est-à-dire au Plan d'ajustement structurel dont les ressorts se sont déjà déployés en Amérique du Sud, en Afrique, ou en Asie du Sud-Est : dévaluer pour relancer les exportations, déréguler le secteur privé et le soumettre à la concurrence étrangère afin d'améliorer sa productivité, contrôler le déficit budgétaire en restreignant les dépenses publiques, privatiser. 


\section{L'Inde de Lakshmi (1993-2002)}

Durant cette décennie, le taux moyen de croissance se hisse à $6 \%$ avec un secteur des services en forte expansion. Par contre, la croissance de l'agriculture décline alors que ses actifs continuent d'augmenter: le revenu moyen du travail agricole croît finalement quatre fois moins vite que le PIB par habitant, une moyenne masquant de fortes disparités (figure 3, tableaux 1 et 2 ).

La page du socialisme nehruvien est définitivement tournée. Il ne s'agit plus d'élever au même rythme le niveau de vie de l'ensemble de la population quitte à ne pas décoller du «taux de croissance hindou », mais de pleinement parier sur « l'effet de percolation » (thèse du trickle down), autrement dit sur une diffusion « vers le bas » des bénéfices d'une activité économique tirée «d'en haut» par deux grands moteurs de croissance : d'une part la consommation de la classe moyenne sur le marché domestique (estimée à l'époque à 200 millions d'individus), d'autre part la vente de services sur le marché international (technologies de l'information entre autres). À la Chine "l'usine du monde », à l'Inde «le bureau». Les grandes villes indiennes grossissent et se transforment alors comme jamais auparavant, celles de Bangalore et d'Hyderabad dans l'Inde du Sud devenant de véritables plateformes informatiques mondiales. McDonald, Domino's Pizza et autres enseignes étrangères se multiplient aux coins des rues, de même qu'explosent le parc de voitures et le prix de l'immobilier urbain.

La libéralisation du système agricole et alimentaire hérité de la Révolution verte est bien plus modeste (Dorin et Landy, 2002) si l'on excepte le secteur du crédit et celui des semences (réformes engagées dès les années 1980). Et avec la signature en 1994 à Marrakech des accords internationaux sur l'agriculture du GATT (dont l'Inde est membre fondateur dès 1947), l'Union entend mieux accéder à certains marchés extérieurs plutôt qu'ouvrir et réformer le sien (Dorin, 2001). Avec une agriculture globalement taxée selon la Mesure globale de soutien, les subventions aux intrants peuvent subsister malgré leur importance financière et tous leurs effets pervers. Les soutiens indirects aux exportations s'affirment mais demeurent bien inférieurs aux équivalents américains ou européens. Enfin, le démantèlement des barrières non tarifaires s'achève en avril 2001, mais elles peuvent être remplacées par un jeu de droits de douane faibles ou élevés selon les produits. Finalement, en amont, malgré diverses tentatives de réduction des subventions aux intrants engagées dès 1991, les prix des engrais et de l'électricité demeurent quasiment stables durant la décennie ce qui, compte tenu de l'inflation, équivaut à subventionner de façon croissante les agriculteurs, du moins ceux qui utilisent abondamment ces intrants. En aval, les réformes successives du PDS s'avèrent plus affirmées mais conduisent à un transfert de charges aux États ainsi qu'aux accumulations et gaspillages des stocks publics. Le cours des céréales s'émancipe (à la hausse) de l'indice général des prix, et plus encore bien d'autres denrées alimentaires (pois et lentilles, légumes et fruits...) à l'exception des huiles végétales que l'Inde réimporte massivement. Jusqu'en 2002, le taux de croissance agricole demeure au plus bas et quelques mois plus tard, en mai 2004, face au Congress, le BJP (parti nationaliste hindou) perd les élections avec un slogan bien malvenu pour la base électorale : si «l'Inde brille ( Shining India) désormais de millionnaires et même de milliardaires en dollars, ce n'est certainement pas dans les campagnes agricoles.

\section{De la croissance sans emploi au droit à l'emploi (2003-2014)}

Depuis 2003, le taux de croissance agricole repart à la hausse alors que la croissance générale culmine à 9,6\% en 2006. Depuis 
2004 surtout, la part de l'agriculture tombe en dessous de $20 \%$ du PIB (14\% en 2013) alors que celle de l'industrie, généralement plus pourvoyeuse d'emplois que les services (67\% en 2013), stagne aux alentours de $20 \%$ depuis le milieu des années 1980 . Le PIB agricole par actif agricole croît en moyenne de $3 \%$ par an, soit trois fois plus vite qu'au cours de la période précédente, mais encore deux fois moins vite que le PIB par habitant (tableau 1).

Cette dernière période est marquée par des interrogations grandissantes sur la «croissance sans emploi » (Raveendran et Kannan, 2009 ; EPW, 2010) et le creusement des inégalités qu'elle engendre. Certes, la pauvreté extrême recule (environ 400 millions d'individus en 2010 avec moins de 1,25 US\$-PPP selon la Banque mondiale), mais celle-ci demeure largement concentrée en zone rurale $(80 \%$ en 2012 selon la Reserve Bank of India) où les bénéfices liés au développement des emplois non agricoles sont pondérés par la plus grande précarité de ces derniers (Himanshu, 2010 ; Himanshu et al., 2013). Les médias indiens et internationaux parlent quant à eux de « crise agraire » en rapportant le suicide de milliers d'agriculteurs surendettés dans certaines régions (plus de 160000 entre 1997 et 2006 selon Nagaraj, 2008). En 2004, la Commission nationale qui conduira à la Politique nationale pour les agriculteurs (NPF) de 2007 entend stimuler une croissance agricole plus rapide et surtout plus profitable et inclusive en termes de revenu, en exceptant néanmoins implicitement des agriculteurs le très large contingent des ouvriers agricoles à temps complet ou partiel (figure 4). En 2005 surtout, le parlement vote, non sans vifs débats, y compris académiques, le National Rural Employment Guarrantee Act (NREGA) ${ }^{13}$, autrement dit un droit par

13. Plus tard renommé le Mahatma Gandhi National Rural Employment Guarantee Act (MGNREGA). famille rurale à 100 jours de travail par an sur des chantiers d'intérêt public (construction de routes, réservoirs, etc.). Sur la base du volontariat, et malgré de nombreux dysfonctionnements, ce «plus grand et ambitieux programme au monde de sécurité sociale et de travaux publics » (IDFC, 2013) connaît un succès et soulage en particulier les agriculteurs avec très peu de terre (Ranaware et al., 2015), ou bien sans (plus de $40 \%$ des ménages ruraux en 2003 selon Rawal, 2008) ${ }^{14}$. Les mailles de ce filet de lutte contre la grande pauvreté et les migrations de détresse seront resserrées en 2013 par le Right to Food Act (ou National Food Security Act) qui étend aux deux tiers de la population indienne le droit d'accès à divers programmes de sécurité alimentaire comme le PDS, le MDMP ou l'ICDS.

Pour notre part, depuis six décennies, nous constatons un déclin de l'agriculture dans le PIB beaucoup plus rapide que dans la population active; la forte croissance du PIB moyen par habitant est en réalité bien mal répartie puisque la majeure partie de la population n'en profite guère. Selon le dernier recensement général (2011), 263 millions d'individus sont en effet encore employés plus de six mois par an dans l'agriculture où le revenu moyen progresse bien moins qu'ailleurs, soit $55 \%$ de la force de travail, des estimations cohérentes avec celles de la FAO (figure 4) et conduisant à évaluer à moins de 0,65 ha la surface moyenne cultivée par actif (près du double en 1960). Pour la première fois depuis l'indépendance de l'Inde, le nombre d'ouvriers agricoles (144 millions) aurait dépassé celui des cultivators (119 millions : agriculteurs cultivant une terre en faire-valoir direct ou indirect, métayage compris). Une autre source indienne (NSSO) estime cependant à « seulement »

14. D'après IDFC (2013), et selon les données des NSS (National Sample Surveys), la part de l'emploi non agricole en zones rurales ne serait passée que de $22 \%$ en 1993/94 à $32 \%$ en 2009/10. 


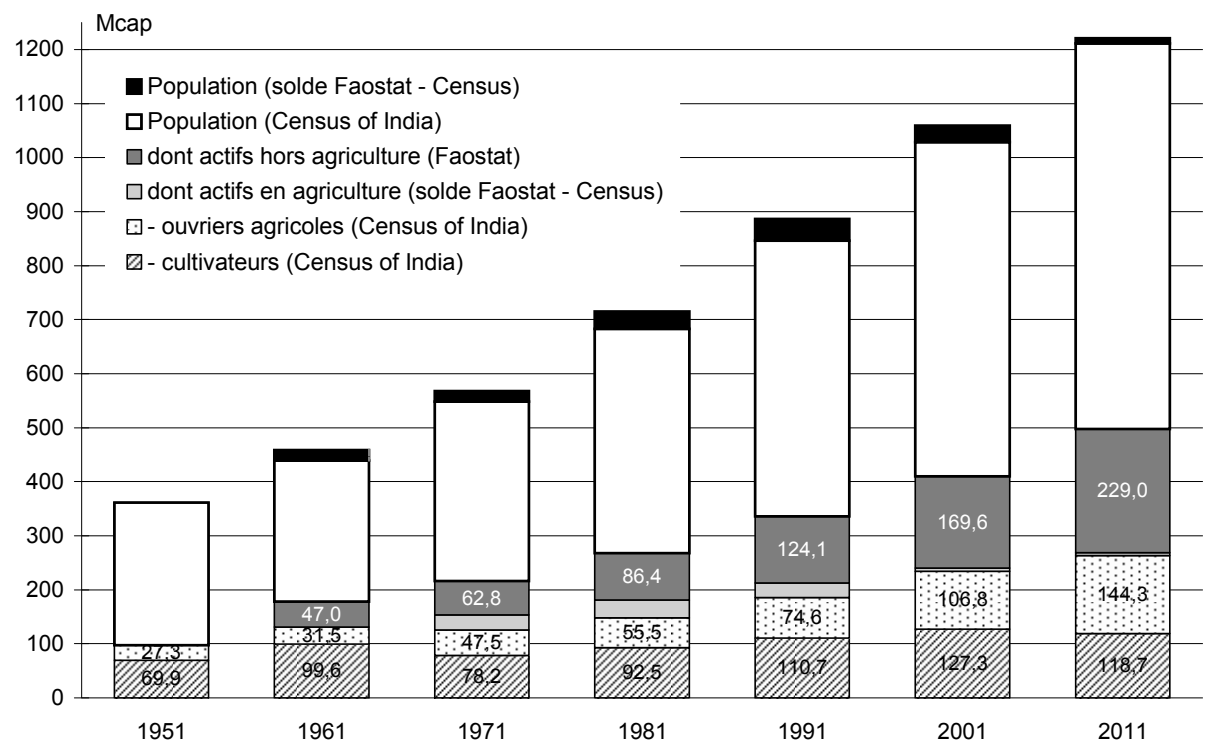

Note : Les données Faostat ne remontent pas avant 1961 et sont extrapolées de 1961 à 1979 avec d'anciennes séries, selon la méthode exposée dans Dorin et al. (2013).

Source des données : Census of India (Government of India, various reports) et Faostat (faostat.fao.org, 26/03/2015)

224 millions le nombre total d'actifs agricoles en 2011-2012, soit $47 \%$ de la force de travail. Ce nombre aurait en effet décru pour la première fois entre 2005 et 2009 pour atteindre aujourd'hui le niveau du début des années 1990 (figure 5), décroissance qui serait liée à la moindre participation féminine dans l'emploi, agricole ou non (Chowdhury, 2011). Une troisième source, le recensement agricole, estime quant à elle que le nombre d'exploitations à considérablement augmenté entre 2000 (120 millions) et 2010 (près de 138 millions) (figure 6a), ceci au prix d'une poursuite accélérée de la réduction de leur taille. En 2010, la surface moyenne des fermes serait de 1,16 ha, avec $67 \%$ d'entre elles inférieures à l'hectare (figure $6 b$ ) alors qu'elles ne cultivent que $22 \%$ de la surface agricole (figure 6c).

La disponibilité en terre par actif agricole est donc extrêmement faible et diminue même au lieu de fortement augmenter comme dans les pays développés (Dorin et al., 2013), ceci en raison d'une croissance de la population active et, surtout, d'une migration largement insuffisante d'emplois en dehors d'une agriculture qui demeure in fine un moindre mal pour beaucoup. Dans un tel contexte, l'augmentation de la productivité du travail agricole (ou simplement son maintien quand la disponibilité en terre diminue) dépend uniquement de l'augmentation de la productivité de la terre ${ }^{15}$. Depuis plus d'un demi-siècle, cette dernière repose en occident comme en orient sur les technologies de la Révolution verte, diffusées et encouragées

15. Comme nous l'avons montré ailleurs (Dorin et al., 2013), l'augmentation de la productivité moyenne du travail agricole dans les pays de l'OCDE (et sa convergence avec la productivité moyenne du travail non agricole) a beaucoup plus reposé sur l'agrandissement des fermes (et leur moto-mécanisation à grande échelle) que sur les augmentations de rendement. 
Figure 5. Population totale et actifs selon NSSO (1972/73 - 2011/12)

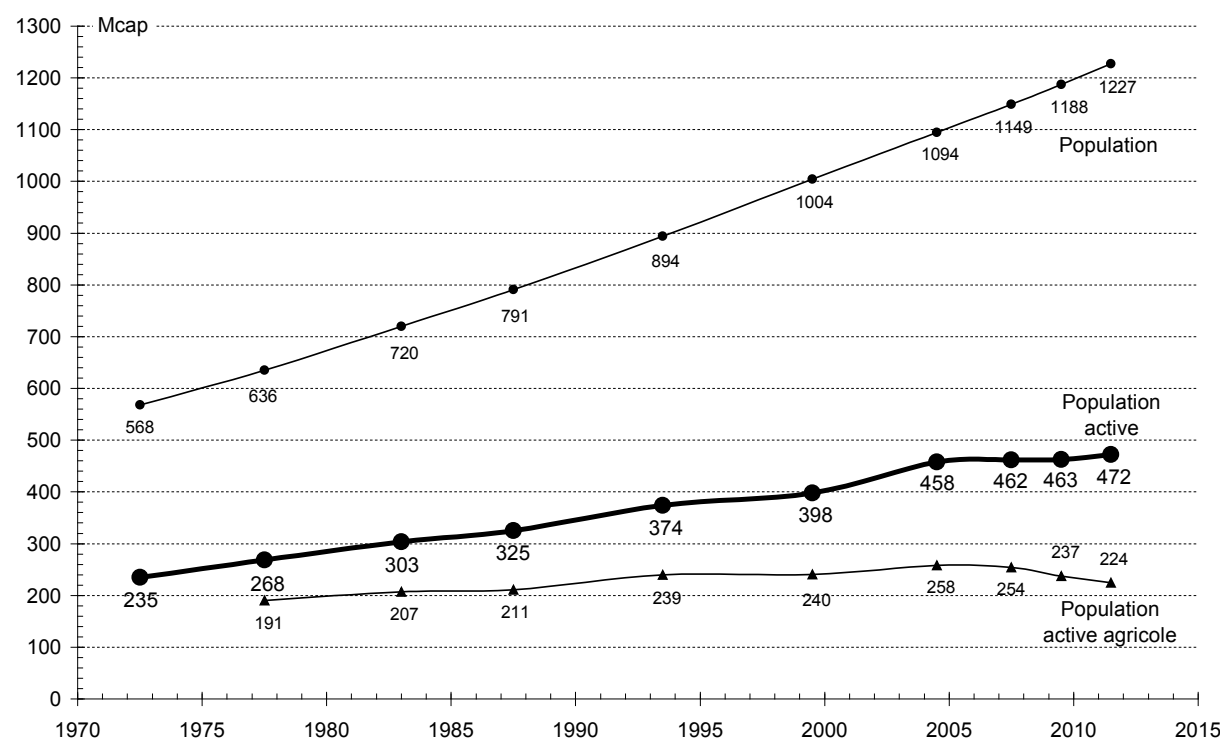

Note : Les données sur les populations actives se rapportent à la comptabilité « PS+SS », i.e. Principal and Subsidiary Status.

Source des données : National Sample Survey Organisation (Government of India, various reports).

Figure 6. Statistiques agricoles par taille d'exploitation, Inde entière (1970-2010)

Nomenclature indienne :

Marginal (marginaux) $:<1$ ha

Small (petits) : 1-2 ha

Medium (moyens) : 4-10 ha

Semi-medium (semi-moyens) : 2-4 ha

Large (grands) : > 10 ha

Figure 6a. Nombre d'exploitations

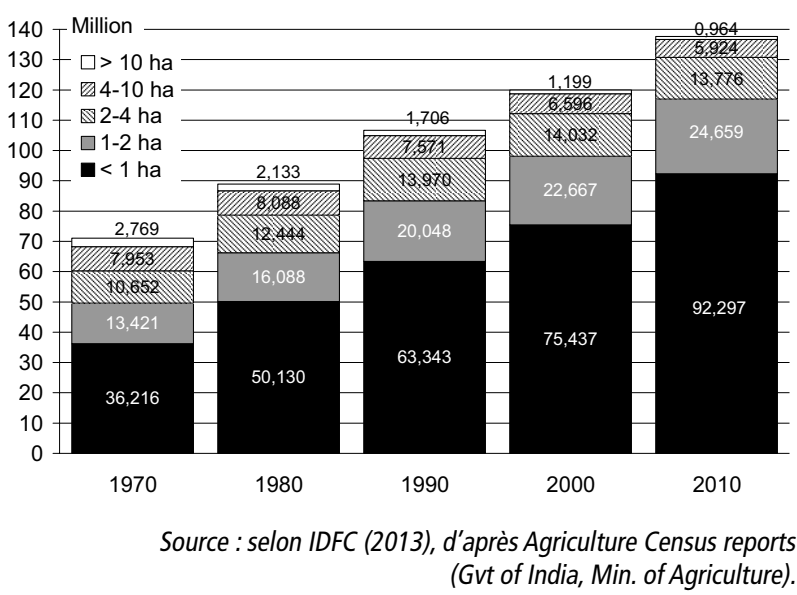

54 • Économie Rurale 352/Mars-Avill 2016 
Figure $6 b$. Surface agricole moyenne par exploitation

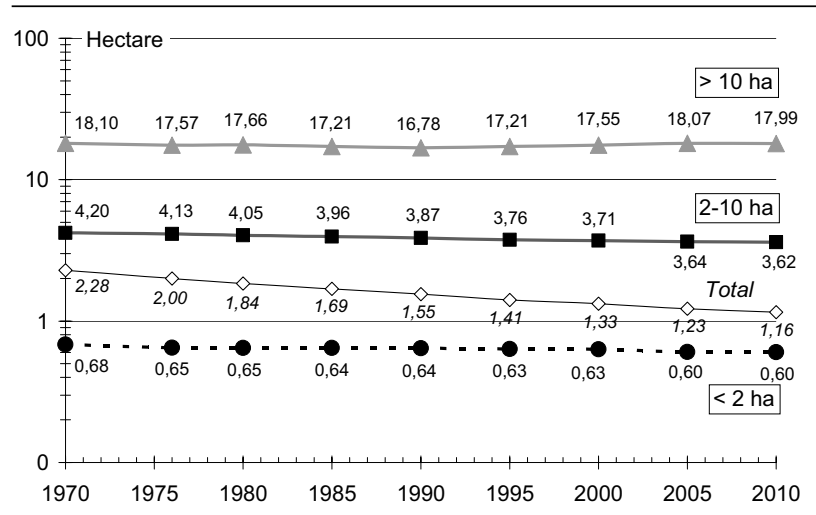

Source : selon selon IDFC (2013) et Kadapatti et Bagalkoti (2014), d'après Agriculture Census reports (Gvt of India, Min. of Agriculture).

Figure $6 c$. Occupation de la surface agricole totale

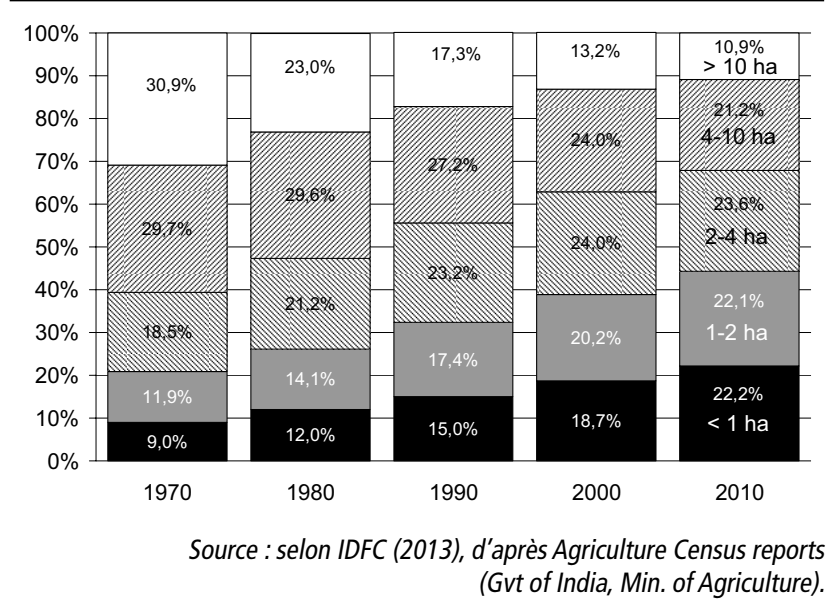

Figure 6d. Irrigation

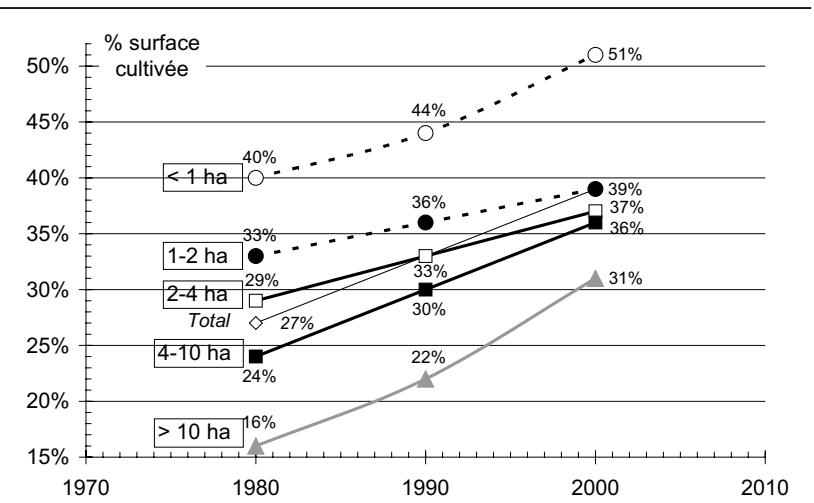

Source : selon Chand et al. (2011), d'après Agriculture Census reports (Gvt of India, Min. of Agriculture). 
Figure 6e. Intensité culturale

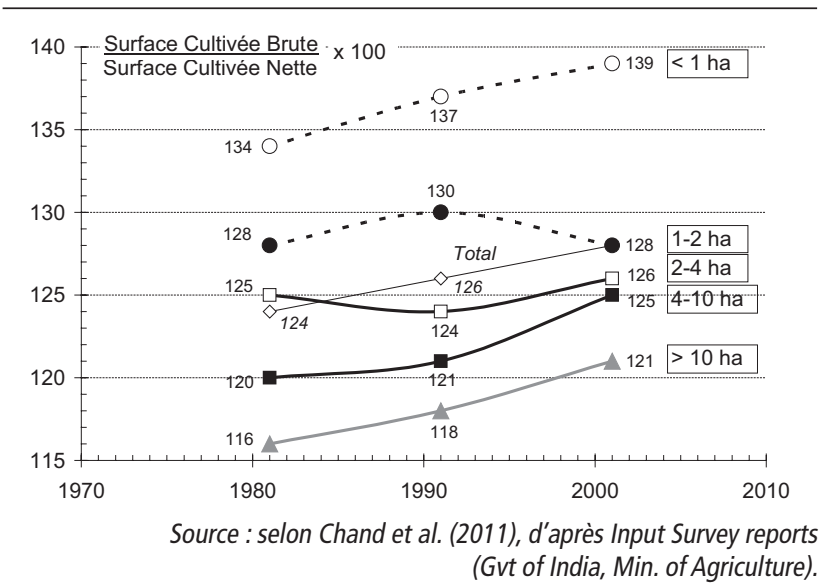

Figure $6 f$. Variétés à haut rendement

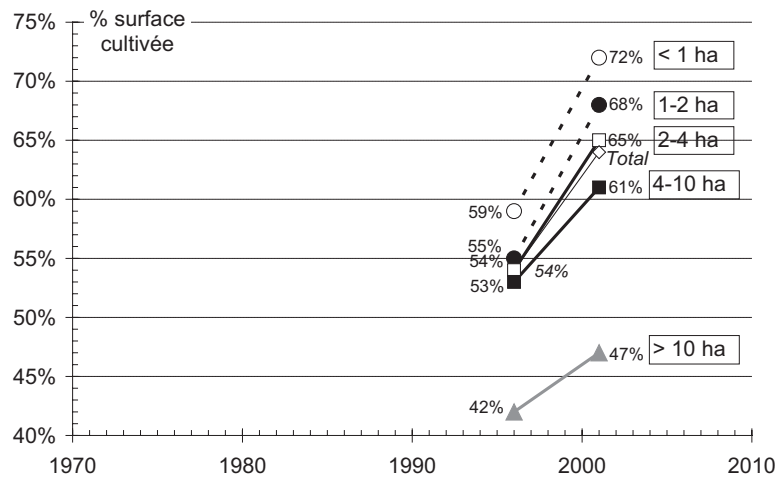

Source : selon Chand et al. (2011), d'après Input Survey reports (Gvt of India,Min. of Agriculture).

Figure 6g. Engrais chimiques

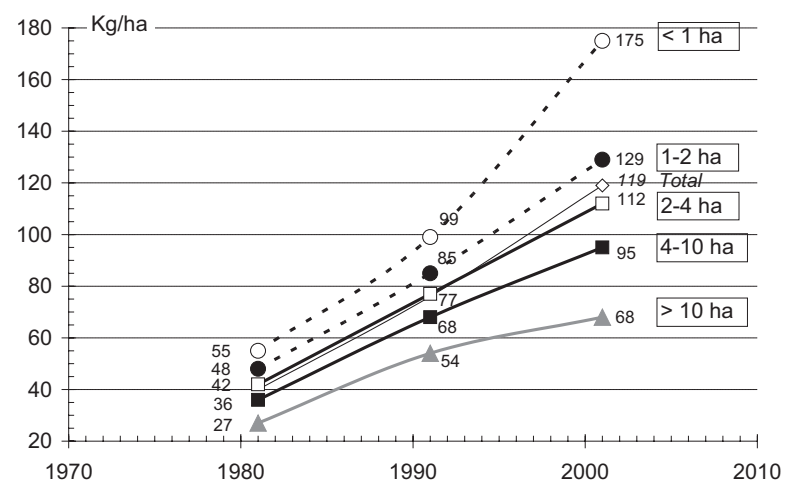

Source : selon Chand et al. (2011), d'après Input Survey reports (Gvt of India, Min. of Agriculture). 
par les communautés tant scientifiques que politiques et industrielles. Indéniablement efficaces à court terme, et fortement subventionnées en Inde, ces technologies ne pouvaient qu'y connaître une adoption grandissante, notamment par ceux de plus en plus faiblement dotés en terre, que ce soit pour l'irrigation (figure $6 d$ ), les variétés de laboratoire (figure $6 f$ ) ou encore les engrais chimiques (figure 6e). Dans cette course au rendement pour compenser la petitesse et la fragmentation des disponibilités foncières, s'affirme alors non seulement le surendettement (auprès de banques si possession de terre, de prêteurs privés sinon) mais aussi une grave érosion des ressources naturelles (sol, eau, biodiversité...) sur lesquelles repose pourtant la productivité de la terre et du travail à long terme. Sans changement de paradigme, tant institutionnel que scientifique et technologique, la sortie de ce piège paraît compromise.

\section{Les vicissitudes de la transition agraire dans deux cantons ruraux du Gujarat}

En complément des analyses macroéconomiques développées dans les deux premières parties, cette troisième section s'intéresse aux transformations en cours à l'échelle du canton. Elle permet une analyse plus fine des changements techniques en cours, des relations sociales opérant à cette échelle, et des inégalités de revenu qui en résultent.

\section{Deux cantons ruraux dans un État modèle de croissance}

Entrons donc maintenant au cœur de deux systèmes agraires du Gujarat (Lehoux, 2014 ; Lucas, 2014 ; Aubron et al., 2015), État du Nord-Ouest de l'Inde qui connaît une forte croissance économique depuis deux décennies (Bagchi et al., 2005 ; Dixit, 2009). Cette dynamique et les réformes qui la sous-tendent lui valent
Figure 7. Petlad et Dharampur dans l'État du Gujarat

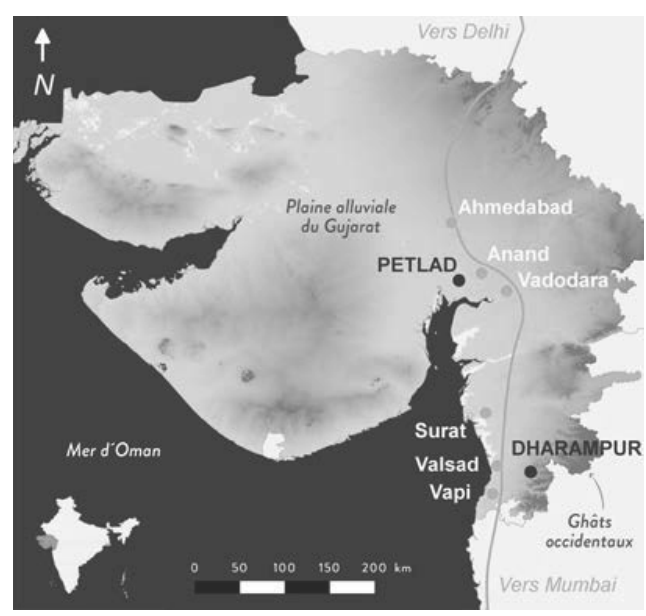

Source : Aubron et al. (2015).

souvent d'être érigé en modèle de croissance et d'émergence à même d'inspirer le restant de l'Inde : une transition agraire plus canonique que celle décrite aux chapitres précédents s'y affirmerait-elle ?

Le canton de Petlad, au nord (figure 7), présente très peu de relief ; c'est une plaine alluviale aujourd'hui quasi intégralement cultivée et irriguée. Plus au sud, le canton de Dharampur, aux possibilités d'irrigation plus réduites, est situé à la frontière entre une plaine irriguée à l'ouest et les montagnes bordant le plateau du Deccan à l'est. Les deux cantons sont soumis au régime des moussons à partir du mois de juin, mais Petlad est trois fois moins arrosé que Dharampur. Ils sont aujourd'hui organisés autour d'une petite ville qui rassemble 30000 à 60000 habitants, et sont situés à proximité du principal axe routier et ferroviaire joignant Delhi à Mumbai. Les centres industriels de Vadodara et d'Ahmedabad, assez anciens et à la croissance économique aujourd'hui ralentie, sont à moins de $70 \mathrm{~km}$ de Petlad. La zone de Dharampur est très proche de Surat et Vapi, dont les activités industrielles sont en pleine expansion, notamment dans le secteur pétrochimique. 
Caractéristique du Nord de l'Inde, l'agriculture de Petlad a longtemps eu le mil pour principale culture, associé à des légumineuses, auxquels s'ajoutait du riz dans les bas-fonds, tout cela en période de mousson. Le foncier de cette plaine fertile a été accaparé de longue date par la caste des Patels, qui mobilisaient la force de travail de castes au statut social inférieur pour mettre en valeur leurs terres. Les Patels sont à l'origine de l'introduction de l'irrigation. Ils l'utilisaient principalement pour la culture du tabac, qui était limitée aux abords des puits jusqu'à la moitié du $\mathrm{XX}^{\mathrm{e}}$ siècle $^{16}$.

Pendant longtemps, les seuls habitants du canton Sud (Dharampur) étaient des Adivasis (tribaux) qui pratiquaient la chasse et la cueillette dans la forêt qui constituait à l'époque leur environnement. Ils maîtrisaient l'abattis-brûlis et l'agroforesterie qui leur permettaient de cultiver du riz ainsi que de l'éleusine, du sorgho et du mil. C'est au XIII' siècle qu'une famille de Rajputs originaire du Rajasthan installa un petit royaume dans la plaine de Dharampur, créant de grandes propriétés confiées à des familles de haute caste et repoussant les populations tribales vers les montagnes. Après déforestation, ces propriétaires de la plaine la firent cultiver chaque année avec du riz et des légumineuses, en période de mousson et en s'appuyant sur une maind'œuvre Adivasi servile (Breman, 2007b).

Dans la plaine des deux cantons, l'élevage, conduit par des castes de pasteurs, jouait un rôle majeur pour renouveler la fertilité des sols cultivés et comme force de traction pour le travail du sol et le transport.

\section{Réforme agraire, partages successoraux et Révolutions verte et blanche}

Dans les deux cantons, les surfaces cultivées par exploitation se sont fortement réduites depuis 1950 : d'après les entretiens

16. Près de $15 \%$ de la surface cultivée sont irrigués en 1950, par des puits de $25 \mathrm{~m}$ de profondeur. que nous avons conduits, elles ont été au moins divisées par deux et jusqu'à dix dans certains cas. Les réformes agraires ont contribué à cette réduction de taille, en démantelant certaines grandes propriétés (de cinq à une centaine d'hectares de terre cultivée) pour grossir le nombre de petites propriétés alors dotées d'un demi à quatre hectares. L'impact de ces réformes agraires sur l'évolution de la structure foncière ne doit toutefois pas être surestimé : comme ailleurs en Inde, une partie des grandes propriétés est parvenue à échapper à la redistribution foncière et certains bénéficiaires de la réforme agraire, faute de moyens pour les cultiver et souvent endettés, ont ensuite revendu les terres reçues à leurs anciens propriétaires (Pouchepadass, 2006 ; Breman, 2007a). C'est bien la division des parcelles entre les fils d'une famille à chaque génération qui, sous l'effet combiné de la croissance démographique et du faible taux de sortie du secteur agricole en termes d'emploi, explique la réduction continue de la taille des exploitations dans les deux zones. Dans le canton de Petlad, un seuil de 0,08 hectare minimum par famille semble toutefois émerger, seuil en deçà duquel la terre n'est héritée que par un des fils, les autres rejoignant le rang des sans-terres (Lucas, 2014). Dans ce contexte de réduction des ressources foncières disponibles par actif agricole, quelles sont les voies d'intensification explorées ?

À partir des années 1970, la Révolution verte et ses trois ingrédients - irrigation, engrais chimiques et variétés à haut rendement - transforment l'agriculture des deux espaces de plaine. Dans le canton de Petlad, les parcelles accueillent généralement aujourd'hui deux cycles de culture par an, et même parfois trois. Les systèmes de culture se sont réorganisés autour du tabac, culture irriguée d'hiver très rémunératrice. Dans la plaine de Dharampur, la double culture annuelle riz/sorgho est la règle dans les petites et moyennes exploitations. Les plus grandes se sont tournées 
vers la canne à sucre, et plus récemment les vergers de manguiers, alors que les toutes petites (moins d'un hectare) développent le maraîchage. Avec l'émergence du modèle coopératif laitier AMUL $^{17}$ et la fourniture d'intrants d'élevage à bas prix (insémination artificielle, concentrés), l'élevage n'est pas en reste dans cette dynamique d'intensification: presque tous les agriculteurs de la plaine dans les deux zones sont devenus producteurs de lait, y compris ceux n'ayant pas de terre. La très grande majorité des élevages compte moins de quatre vaches ou bufflesses, mais certains ont investi dans de plus grands élevages rassemblant 30 à 200 mères. Tout ou partie des céréales, mais aussi du lait, sont autoconsommés par les familles d'agriculteurs, en particulier dans les petites exploitations.

L'accroissement des productions par unité de surface permis par les Révolutions verte (des céréales) et blanche (du lait) estil à même de contrebalancer durablement la diminution de la surface disponible par exploitation? Trois éléments suggèrent une réponse négative. Tout d'abord, si cet accroissement s'est bien produit depuis 1970, on atteint aujourd'hui un palier tant en termes d'expansion de l'irrigation à de nouvelles surfaces, en particulier dans le canton de Petlad, que d'augmentation des rendements. La production laitière, quant à elle, a profité des résidus de culture abondants fournis par la Révolution verte, mais appuie maintenant sa croissance sur des aliments produits pour partie à l'extérieur du territoire. En second lieu, ces formes d'intensification sont toutes deux problématiques pour l'environnement: comme

17. AMUL est la première coopérative laitière créée dans le Gujarat en 1946 à proximité du canton de Petlad. Le modèle sera ensuite repris et diffusé ailleurs en Inde par le National Dairy Development Board (NDDB) au cours de l'Operation Flood, ensemble de politiques mises en œuvre entre 1970 et 1996 et en partie financées par la revente de l'aide alimentaire laitière (Dorin et Landy, 2002 ; Scholten, 2010). dans la plupart des zones irriguées du souscontinent, les ressources hydriques utilisées pour l'irrigation dans les deux cantons correspondent pour l'essentiel à de l'eau souterraine, prélevée via des forages privés échappant à toute régulation publique (Shah, 2010). Si les deux espaces ne figurent pas parmi les zones du pays les plus menacées à court terme par la diminution des ressources en eau (Roy et Shah, 2002), le niveau des nappes phréatiques tend néanmoins à baisser, et la profondeur des forages à s'accroître (60 m dans le canton de Petlad et jusqu'à 100 m à Dharampur). En outre, la forte densité d'animaux dans les deux plaines, nourris avec des concentrés et fourrages produits en partie à l'extérieur du territoire, génère des excédents minéraux pouvant renforcer des pollutions liées à des surconsommations d'engrais chimiques. Enfin, si l'agriculture de ces deux cantons est assez peu moto-mécanisée - des tracteurs et motoculteurs sont utilisés principalement pour le travail du sol -, elle consomme par contre beaucoup d'énergie fossile pour l'irrigation et la fabrication intrants (engrais surtout), ce qui vient indirectement abonder l'émission importante de gaz à effet de serre par l'élevage et la fertilisation. La troisième limite des Révolutions verte et blanche est liée à leur cantonnement aux espaces irrigués : les habitants de la zone de montagne du canton de Dharampur sont ainsi restés à l'écart des gains de productivité obtenus dans la plaine et cultivent toujours le riz, des légumineuses et de l'éleusine à partir des seules pluies de mousson. La coopérative laitière y a appuyé l'installation de centres de collecte mais la faible disponibilité en biomasse fourragère limite fortement les volumes de lait produits.

\section{Inégales répartitions de terre, d'eau et de valeur ajoutée}

La transition agraire dans ces deux cantons, déjà fortement contrainte aux plans structurel et technique comme nous venons de 
l'exposer, paraît encore plus compromise si l'on intègre les inégalités sociales dans le champ d'analyse. En effet, les ressources foncières par actif agricole sont faibles en moyenne, mais aussi très inégalement réparties. Dans le canton de Petlad, les propriétaires Patel, qui ont pour certains migré à l'étranger (États-Unis notamment) tout en conservant leurs droits fonciers, possèdent jusqu'à 10 ha, alors que l'essentiel des actifs agricoles sont, soit des petits propriétaires de 0,1 à 0,4 ha, soit des sans-terres travaillant comme métayers ou journaliers. D'après le recensement de 2011, plus de la moitié de la population active agricole $\mathrm{du}$ district d'Anand (auquel appartient le canton de Petlad) sont des travailleurs sans terre restés à l'écart du processus de réforme agraire. Dans le canton Sud, une poignée de producteurs de mangue possèdent jusqu'à 40 ha par exploitation là où la plupart des fermes exploitent 0,5 à 4 ha. Ces inégalités foncières se doublent d'un inégal accès à l'eau d'irrigation, qui a deux origines : la première est liée à l'éloignement des nappes souterraines, expliquant par exemple que les Adivasis des montagnes du canton Sud de Dharampur ne peuvent cultiver leurs terres qu'en période de mousson. La seconde est liée aux capacités différenciées des familles, sur un même espace, à investir dans des équipements d'irrigation. Ainsi, bon nombre de petits propriétaires du canton Nord ne possèdent pas de forage et achètent l'eau aux plus grands propriétaires fonciers, mieux équipés. Dans le canton Sud, les agriculteurs qui n'ont pas les moyens d'investir dans les forages profonds manquent alors d'eau pour la canne à sucre, le maraîchage ou les manguiers, et ne produisent alors que riz et sorgho à moindre valeur ajoutée.

Par ailleurs, les inégalités ne se résument pas, dans les deux cantons, à des inégalités d'accès à ces deux ressources clés que sont la terre et l'eau. Elles découlent également des modalités de répartition de la valeur ajoutée dans le cadre des relations de métayage ou de salariat qui, ici comme ailleurs en Inde (Rawal, 2006 ; Breman, 2007b ; Ramachandran et al., 2010), sont très favorables aux propriétaires fonciers. Ces relations sont structurantes des deux systèmes agraires puisque presque tous les propriétaires en zone irriguée font appel à de la main-d'œuvre extérieure à la famille pour conduire tout ou partie de leurs cultures ou élevage. Pratiqué dans les deux cantons, le salariat agricole est parfois permanent (cas des élevages avec plus de 12 têtes), saisonnier (pour la récolte des mangues ou du riz à Dharampur par exemple) ou le plus souvent journalier. Les travailleurs salariés sont les Adivasis des montagnes dans le canton Sud, les sans-terres les plus pauvres dans le canton Nord, auxquels s'ajoutent, pour certains travaux, des salariés en provenance de régions plus pauvres de l'Inde. Notre évaluation de la valeur ajoutée moyenne par jour de travail $^{18}$ pour les principaux systèmes de culture pratiqués dans les deux zones irriguées montre que celle-ci est deux à cinq fois supérieure au salaire agricole journalier (Aubron et al., 2015). Les propriétaires récupèrent ainsi la majeure partie de la richesse créée, pour une contribution au travail souvent minime (dans certains cas elle n'atteint pas $5 \%$ du temps de travail). Les accords de métayage, qui n'est pratiqué que dans le canton de Petlad, ne sont pas systématiquement plus favorables au travailleur, ici le métayer. Néanmoins, le métayage est préféré au salariat quand c'est possible, parce qu'il inclut souvent d'autres avantages, tels que des prêts

18. La valeur ajoutée (produit brut - incluant le cas échéant les produits autoconsommés - auquel on soustrait les consommations intermédiaires) est rapportée au nombre de journées de travail nécessaire à sa création, pour chaque système de culture. Nous comparons ici cette productivité du travail avec le salaire agricole journalier, qui était de 100 à 150 roupies par jour $(1,2$ à $2 €)$ au moment de l'étude. 
accordés par le propriétaire ou le droit de cultiver des fourrages sur le bord des champs. Les accords de métayage ne sont conclus qu'entre certaines castes et certaines familles, et n'est donc pas métayer qui veut parmi la masse de sans-terres du canton Nord.

L'emploi non agricole, vers lequel les regards se tournent sans cesse comme voie de sortie de la pauvreté, ne semble guère rebattre les cartes de ce jeu social d'apparence très inégalitaire. Comme ailleurs en Inde (Abraham, 2009 ; Himanshu et al., 2013), les activités non agricoles des deux cantons ont pourtant bien connu un essor au cours des dernières décennies. Il apparaît néanmoins que l'activité non agricole mise en œuvre est étroitement dépendante de la position sociale au sein du système agraire: certains propriétaires fonciers Patel du canton Nord occupent ainsi un emploi salarié à l'étranger, et les autres sont souvent à la tête de petites entreprises locales (restaurants et lieux de réception pour des mariages, entreprise de fabrication de statues, etc.). Les producteurs de canne à sucre et de mangues du canton Sud ont généralement un emploi salarié dans le secteur industriel ou tertiaire des villes en croissance du Sud du Gujarat. Mais pour les journaliers et les métayers de Petlad comme pour les Adivasis des montagnes de Dharampur, il est quasiment impossible de trouver des emplois autrement qu'à la journée, très physiques, mal rémunérés et précaires même durant les mois agricoles creux. Ils vendent leur force de travail à l'échelle locale dans le secteur des services, de la construction et des travaux publics et peinent à explorer les marchés du travail plus lointains en raison du coût du transport et du logement. Ces plus démunis, qui représentent d'après nos estimations au moins un tiers de la population soit plus de 100000 personnes pour les deux cantons, restent donc extrêmement dépendants de l'offre d'emplois agricoles locaux pour assurer leur subsistance.
*

* *

Vingt ans après le plan d'ajustement de 1991, l'économie indienne est plus que jamais tournée vers les marchés non agricoles tant domestiques qu'internationaux, et sa croissance frôle $9 \%$, un taux bien supérieur à celui de sa démographie et sa population active. Une telle dynamique devrait a priori entraîner la sortie de l'agriculture d'un grand nombre d'actifs vivant pauvrement de cette activité. Comme nous l'avons cependant montré en première analyse, ce schéma de croissance peine grandement à s'affirmer en Inde : la population active agricole continue d'augmenter en nombre au lieu de régresser, et son écart de revenu avec les autres actifs s'accentue au lieu de se résorber malgré des révolutions d'intensification de la productivité de la terre engagées dès les années 1960. Cette évolution, à l'antipode de l'expérience simultanée des pays riches, mais partagée par la majorité des pays d'Asie continentale, pose une question légitime : est-ce un avatar qui disparaîtra avec le temps, après des progrès d'éducation et d'adaptation des compétences aux évolutions de marchés, ou bien l'expression des limites du modèle de convergence vers « un monde sans agriculture » dont le déploiement resterait finalement peu ou prou confiné aux espaces humains et fenêtres historiques qui l'ont porté ? Notamment parce que l'intensité capitalistique croissante de l'économie ne permet plus d'absorber, comme aux temps de la «manufacture », des centaines de millions d'actifs dans le seul cas indien (Dorin et al., 2013) ?

Comme nous l'avons montré en seconde analyse, les dirigeants de l'Inde indépendante ont en tout cas cherché pendant plus de trois décennies une troisième voie de développement susceptible de contenir les divergences de revenu, notamment entre villes et campagnes. Mais crises et évolutions des contextes tant domestique 
qu'international ont sonné le glas dès les années 1980 de cette «économie mixte » d'inspiration nehruvienne, voire gandhienne avec l'encouragement des petites industries villageoises. Le changement de paradigme économique - officialisé en 1991 - conduit à une ouverture au monde sans précédent de l'Union et à de profondes mutations internes. Mais l'Inde ne brille alors guère que par l'extraordinaire multiplication de ses millionnaires, et sa «croissance sans emploi » conduit à instaurer en 2005 un droit par famille rurale à 100 jours de travail par an sur des chantiers d'intérêt public.

Les diagnostics agraires conduits dans deux cantons du Gujarat confirment en troisième analyse le caractère problématique de la transition agraire même dans un État indien à croissance économique des plus dynamiques. Ils mettent notamment en évidence que l'accroissement sans précédent de la productivité de la terre grâce aux Révolutions verte et blanche ne permet pas de compenser durablement la contraction des ressources foncières par actif agricole. Les impacts environnementaux tant locaux que globaux de cette intensification posent par ailleurs de graves questions. Enfin, les sociétés rurales étudiées apparaissent elles-mêmes structurées par de très inégaux accès à la terre, à l'eau, mais aussi à la valeur ajoutée compte tenu des accords de salariat ou métayage en vigueur.

L'ensemble de notre exposé interroge autant la reproductibilité de la transformation structurelle expérimentée par les pays riches, que le projet de croissance inclusive et de plus grande équité qui a animé l'Inde depuis son indépendance.
La réelle capacité de ce pays à l'innovation technique et institutionnelle est donc plus que jamais réinterpellée, autant pour elle-même, ses ressources humaines et naturelles, que pour bien d'autres espaces qui partagent (ou partageront bientôt) des problématiques similaires de développement (sur le cas africain, voir Losch et al., 2013 ; Dorin, 2014). À moins d'envisager une aussi libre circulation internationale de la main-d'œuvre que celle du capital aujourd'hui, on peut en effet parier que la croissance des inégalités qui a pu accompagner par le passé des périodes de forte croissance (Kuznets, 1966) ait désormais bien du mal à se résorber. La place de l'agriculture et des agriculteurs dans la croissance et la convergence des revenus est pour cette raison à repenser. Cette place n'est probablement pas uniquement dans la production de commodités « primaires » (aliments, fibres, énergies...), mais aussi dans celle de services «tertiaires", en particulier environnementaux (stockage de carbone et biodiversité, recyclage d'eaux et nutriments, pollinisation, contrôle des épidémies ou inondations, etc.). Ceci sous réserve que ces services soient effectivement rendus et bien sûr rémunérés, ce qui implique en amont de profondes réformes tant institutionnelles que technologiques (Dorin et al., 2013) qui veilleraient aussi à mieux répartir les accès au foncier, à l'eau et à la valeur ajoutée. L'Inde démocratique et scientifique pourrait ici s'affirmer. Il lui reste à en trouver la force politique ainsi que les soutiens financiers pour expérimenter avant d'autres une voie de croissance mondialement plus durable et inclusive que celle d'aujourd'hui. 


\section{RÉFÉRENCES BIBLIOGRAPHIQUES}

Abraham V. (2009). Employment Growth in rural India: Distress-Driven? Economic \& Political Weekly, vol. XLIV, $\mathrm{n}^{\circ} 16$, pp. 97-104.

Appu P. S. (1997). Land reforms in India: a survey of policy, legislation and implementation. New Delhi, Vikas, 363 p.

Aubron C., Lehoux H., Lucas C. (2015). Pauvreté et inégalités en Inde rurale : réflexion à partir de deux diagnostics agraires dans l'État du Gujarat. EchoGéo [En ligne], n 32, pp. 1-17.

Bagchi A. K., Das P., Chattopadhyay S. K. (2005). Growth and Structural Change in the Economy of Gujarat, 1970-2000. Economic \& Political Weekly, vol. XL, $\mathrm{n}^{\circ}$ 28, pp. 3039-3047.

Basole A., Basu D. (2015). Non-Food Expenditures and Consumption Inequality in India. Economic \& Political Weekly, vol. L, $\mathrm{n}^{\circ} 36, \mathrm{pp} .43-53$.

Breman J. (2007a). Labour bondage in west India: from past to present. New Delhi, Oxford University Press, $280 \mathrm{p}$.

Breman J. (2007b). The poverty regime in village India: half a century of work and life at the bottom of the rural economy in South Gujarat. NewDelhi, Oxford University Press, $458 \mathrm{p}$.

Chand R., Prasanna P. A. L., Singh A. (2011). Farm Size and Productivity: Understanding the Strengths of Smallholders and Improving Their Livelihoods. Economic \& Political Weekly, vol. XLVI, n 26-27, pp. 5-11.

Chenery H., Srinivasan T. N. (dir.). (1998). Handbook of Development Economics. Vol. 1, Part 2, Structural Transformation, Eastbourne, Elsevier, pp. 197-465.

Chowdhury S. (2011). Employment in India: What Does the Latest Data Show? Economic \& Political Weekly, vol. XLVI, n 32 , pp. 23-26.

CSO (2015). National Accounts Statistics of India. Central Statistics Office, Government of India, http://dbie.rbi.org.in/DBIE (03/2015).
Datta S., Sharma V. (dir.). (2010). State of India's Livelihoods Report 2010. New Delhi, Sage, 150 p.

Dixit A. K. (2009). Agriculture in a High Growth state: Case of Gujarat (1960 to 2006). Economic \& Political Weekly, vol. XLIV, n ${ }^{\circ}$ 50, pp. 64-71.

Dorin B. (2001). L'Inde dans le commerce agricole international. Conditions et bilan de mise en œuvre des accords de Marrakech. Notes et Études économiques, $\mathrm{n}^{\circ} 13$, pp. 49-84.

Dorin B. (dir.). (2003). The Indian Entrepreneur. A Sociological Profile of Businessmen and their Practices. New Delhi, Manohar, 171 p., https://hal.archives-ouvertes.fr/hal00137619

Dorin B. (2014). Dynamiques agricoles en Afrique subsaharienne : une perspective à 2050 des défis de la transformation structurelle. New Delhi, Centre de Sciences Humaines, 52 p., http://hal.cirad.fr/cirad01113012

Dorin B., Pingault N., Boussard J.-M. (2001). Formation et répartition des gains de productivité agricole en Inde. 1980-1996. Économie rurale, $\mathrm{n}^{\circ} 263$, pp. 78-91.

Dorin B., Landy F. (2002). Agriculture et alimentation de l'Inde. Les vertes années (1947-2001). Paris, INRA, 248 p.

Dorin B., Jullien T. (dir.) (2004). Agricultural Incentives in India. Past Trends and Prospective Paths towards Sustainable Development. New Delhi, Manohar, 334 p.

Dorin B., Hourcade J.-C., Benoit-Cattin M. (2013). A World without Farmers? The Lewis Path Revisited. Nogent-sur-Marne, CIRED, 26 p., https://hal.archives-ouvertes. fr/hal-00866413

EPW (2010). Jobless Growth. Economic \& Political Weekly, vol. XLV, n ${ }^{\circ}$ 39, pp. 7-8.

FAO (2009). The State of Food Insecurity in the World. Economic crises, impacts and lessons learned. Rome, Food and Agricultural Organization of the United Nations, $61 \mathrm{p}$. 
FAO (2010). FAOSTAT, Internet web portal and database. Food and Agricultural Organization of the United Nations, http:// faostat.fao.org/ (26/10/2010).

Gollin D. (2014). The Lewis Model: A 60-Year Retrospective. Journal of Economic Perspectives, vol. $28, \mathrm{n}^{\circ} 3$, pp. 71-88.

Gollin D., Lagakos D., Waugh M. E. (2014). The Agricultural Productivity Gap. Quarterly Journal of Economics, vol. 129, $\mathrm{n}^{\circ} 2$, pp. 939-993.

Herrendorf B., Rogerson R., Valentinyi Á. (2014). Growth and Structural Transformation. In Philippe A., Steven N. D. (dir.), Handbook of Economic Growth, Amsterdam-Boston, Elsevier, vol. 2, pp. 855-941.

Himanshu (2010). Towards New Poverty Lines for India. Economic \& Political Weekly, vol. XLV, $n^{\circ} 1$, pp. 38-48.

Himanshu, Lanjouw P., Murgai R., Stern N. (2013). Nonfarm diversification, poverty, economic mobility, and income inequality: a case study in village India. Agricultural Economics, vol. 44, n ${ }^{\circ}$-5, pp. 461-473.

IDFC (2013). India Rural Development Report 2012/13. Delhi, Orient Blacksawn, 331 p.

Janvry A. D. (2010). Agriculture for development: new paradigm and options for success. Agricultural Economics, vol. 41, pp. 17-36.

Johnston B. F., Mellor J. W. (1961). The Role of Agriculture in Economic Development. The American Economic Review, vol. 51, $\mathrm{n}^{\circ} 4$, pp. 566-593.

Kadapatti R. G., Bagalkoti S. T. (2014). Small Farms and Agricultural Productivity. A Macro Analysis. International Journal of Social Science Studies, vol. 2, $\mathrm{n}^{\circ} 3$, pp. 123-135.

Kuznets S. (1966). Modern Economic Growth: Rate, Structure and Spread. New Haven and London, Yale University Press, xvii-529 p.

Lehoux H. (2014). Diagnostic agraire. Valsad district, Sud du Gujarat, Inde. Montpellier, Montpellier SupAgro.

Lewis W. A. (1954). Economic Development with Unlimited Supplies of Labour. The Manchester School, vol. 22, $\mathrm{n}^{\circ} 2$, pp. 139-191.
Lin J. Y. (2011). From Flying Geese to Leading Dragons: New Opportunities and Strategies for Structural Transformation in Developing Countries. Helsinki, World Institute for Development Economics Research, 45 p.

Losch B., Fréguin-Gresh S., White E. T. (2013). Transformations rurales et développement. Les défis du changement structurel dans un monde globalisé. Montreuil, Pearson, 298 p.

Lucas C. (2014). Diagnostic agraire du taluka de Petlad. Rétrospective, actualité et perspectives d'un bassin d'approvisionnement de la coopérative laitière AMUL. Montpellier, Montpellier SupAgro.

Markovits C. (2014). Aux sources du capitalisme indien. Un entretien avec Jules Naudet. La Vie des idées, http://www.laviedesidees. fr/Aux-sources-du-capitalisme-indien.html, 13/06/2014.

Nagaraj K. (2008). Farmers' Suicides in India: Magnitudes, Trends and Spatial Patterns. Chennai, Bharathi Puthakalayam, 38 p.

Piveteau A., Rougier É. (2010). Émergence, l'économie du développement interpellée. Revue de la régulation. vol. 7। $1^{\mathrm{er}}$ semestre, mis en ligne le 3 juin 2010, http://regulation. revues.org/7734.

Pouchepadass J. (2006). Le monde rural. In C. Jaffrelot (dir.), L'Inde contemporaine de 1950 à nos jours, Paris, Fayard, pp. 421-458.

Raina R. S. (2011). Institutional Strangleholds: Agricultural Science and the State in India. In D. Narayana, R. Mahadevan (dir.), Shaping India: Economic Change in Historical Perspective, New Delhi, Routledge, pp. 99-123.

Ramachandran V. K., Rawal V., Swaminathan M. (2010). Socio-economic surveys of three villages in Andhra Pradesh: A study of agrarian relations. New Delhi, Tulika Books.

Ranaware K., Das U., Kulkarni A., Narayanan S. (2015). MGNREGA Works and Their Impacts. vol. $\mathrm{L}, \mathrm{n}^{\circ} 13$, pp. 53-61.

Raveendran G., Kannan K. P. (2009). Growth sans Employment: A Quarter Century of Jobless Growth in India's Organised Manufacturing. Economic \& Political Weekly, vol. XLIV, n ${ }^{\circ}$ 10, pp. 80-91.

Rawal V. (2006). The Labour Process in Rural Haryana (India): A Field-Report from Two 
Villages. Journal of Agrarian Change, vol. 6, pp. 538-583.

Rawal V. (2008). Ownership Holdings of Land in Rural India: Putting the Record Straight. Economic \& Political Weekly, vol. XLIII, $\mathrm{n}^{\circ} 10$, pp. 43-47.

Rodrik D. (2013). Unconditional Convergence in Manufacturing. Quarterly Journal of Economics, vol. 128, ${ }^{\circ}$ 1, pp. 165-204.

Roy A. D., Shah T. (2002). Socio-ecology of groundwater irrigation in India. In R. Llamas, E. Custodio (dir.), Intensive Use of Groundwater Challenges and Opportunities. CRC Press, pp. 307-335.

Rudolph L. I., Rudolph S. H. (1987). In Pursuit of Lakshmi: The Political Economy of the Indian State. Chicago, University of Chicago Press.

Sauvy A. (1980). La machine et le chômage. Paris, Dunod.
Scholten B. A. (2010). India's White Revolution: Operation Flood, Food Aid and Development. London, I.B. Tauris.

Sen A. (1981). Poverty and Famines: An Essay on Entitlement and Deprivation. Oxford, Clarendon Press, 257 p.

Shah T. (2010). Taming the anarchy: Groundwater governance in South Asia. Routledge.

Subramanian S., Jayaraj D. (2015). Growth and Inequality in the Distribution of India's Consumption Expenditure: 1983 to 2009-10. Economic \& Political Weekly, vol. L, $\mathrm{n}^{\circ} 32$, pp. 39-47.

Timmer C. P. (2009). A World without Agriculture. The Structural Transformation in Historical Perspective. Washington DC, The American Enterprise Institute, 96 p.

UNSTAT (2010). National Accounts Main Aggregate Database. United Nations, Statistical Division, http://unstats.un.org/unsd/ snaama/selbasicFast.asp, 10/11/2010. 